University of Nebraska - Lincoln

DigitalCommons@University of Nebraska - Lincoln

2003

\title{
Indirect evidence of density-dependent population regulation in Aponomma hydrosauri (Acari: Ixodidae), an ectoparasite of reptiles
}

\author{
Andrew J. Tyre \\ University of Nebraska at Lincoln, atyre2@unl.edu \\ C. Michael Bull \\ Flinders University of South Australia,, michael.bull@flinders.edu.au \\ Brigitte Tenhumberg \\ University of Nebraska - Lincoln, btenhumberg2@unl.edu \\ Neil Chilton \\ University of Melbourne
}

Follow this and additional works at: https://digitalcommons.unl.edu/natrespapers

Part of the Natural Resources and Conservation Commons

Tyre, Andrew J.; Bull, C. Michael; Tenhumberg, Brigitte; and Chilton, Neil, "Indirect evidence of densitydependent population regulation in Aponomma hydrosauri (Acari: Ixodidae), an ectoparasite of reptiles" (2003). Papers in Natural Resources. 222.

https://digitalcommons.unl.edu/natrespapers/222

This Article is brought to you for free and open access by the Natural Resources, School of at DigitalCommons@University of Nebraska - Lincoln. It has been accepted for inclusion in Papers in Natural Resources by an authorized administrator of DigitalCommons@University of Nebraska - Lincoln. 


\title{
Indirect evidence of density-dependent population regulation in Aponomma hydrosauri (Acari: Ixodidae), an ectoparasite of reptiles
}

\author{
Andrew J. Tyre, ${ }^{1}$ C. Michael Bull, ${ }^{2}$ Brigitte Tenhumberg, ${ }^{1}$ and Neil Chilton ${ }^{3}$ \\ 1. The Ecology Centre, Departments of Zoology and Entomology and Mathematics, \\ University of Queensland, St. Lucia, Queensland, Australia \\ 2. School of Biological Sciences, Flinders University of South Australia, Adelaide, South Australia, Australia \\ 3. Department of Veterinary Science, University of Melbourne, Werribee, Victoria, Australia \\ Corresponding author - A. J. Tyre. Present address: School of Natural Resource Sciences, University of Nebraska-Lincoln, \\ 202 Natural Resources Hall, East Campus, Lincoln, Nebraska, 68583-0819, USA; email atyre2@unl.edu
}

\begin{abstract}
The extent to which density-dependent processes regulate natural populations is the subject of an ongoing debate. We contribute evidence to this debate showing that density-dependent processes influence the population dynamics of the ectoparasite Aponomma hydrosauri (Acari: Ixodidae), a tick species that infests reptiles in Australia. The first piece of evidence comes from an unusually long-term dataset on the distribution of ticks among individual hosts. If density-dependent processes are influencing either host mortality or vital rates of the parasite population, and those distributions can be approximated with negative binomial distributions, then general host- parasite models predict that the aggregation coefficient of the parasite distribution will increase with the average intensity of infections. We fit negative binomial distributions to the frequency distributions of ticks on hosts, and find that the estimated aggregation coefficient $k$ increases with increasing average tick density. This pattern indirectly implies that one or more vital rates of the tick population must be changing with increasing tick density, because mortality rates of the tick's main host, the sleepy lizard, Tiliqua rugosa, are unaffected by changes in tick burdens. Our second piece of evidence is a re-analysis of experimental data on the attachment success of individual ticks to lizard hosts using generalized linear modeling. The probability of successful engorgement decreases with increasing numbers of ticks attached to a host. This is direct evidence of a density-dependent process that could lead to an increase in the aggregation coefficient of tick distributions described earlier. The population-scale increase in the aggregation coefficient is indirect evidence of a density-dependent process or processes sufficiently strong to produce a population-wide pattern, and thus also likely to influence population regulation. The direct observation of a density-dependent process is evidence of at least part of the responsible mechanism.
\end{abstract}

Keywords: maximum likelihood estimation, negative binomial distribution, population regulation, Tiliqua rugosa

\section{Introduction}

The issue of what forces are responsible for regulating the abundance of organisms has been a source of controversy among ecologists for decades (Peet 1991), and the debate shows no signs of abating (Bonsall et al. 1998; Murray 2000; Tyre \& Tenhumberg 2000). Densitydependent mechanisms must operate at some level of abundance, if only in competition for living space (den Boer 1991). For example, it is not possible for an ectoparasite (e.g. a tick) to reach densities much higher than the surface area of the host divided by the area of the ectoparasite. However, in many systems, density-dependent effects on fecundity or mortality are absent or undetectable at typical densities of natural populations (e.g. Hassell et al. 1987; Roitberg et al. 1990; den Boer 1991; Stiling et al. 1991). Responses of ecological processes such as survival, development and reproduction to changes in conspecific density can be weak, and detection of these effects can also depend on the temporal and spatial scale in question (Ray \& Hastings 1996).

The controversy over the extent to which densitydependent mechanisms regulate populations also applies to parasites. Two recent books on the ecology of parasites make opposing statements about density dependence; Smith (1994) claims that abundant evidence exists for population regulation through density-dependent mechanisms, whereas Esch and Fernández (1993) assert that most parasite populations are regulated by density-independent mechanisms. Parasite fecundity, survival, development and host mortality have all been found to vary with host-parasite burden (Smith 1994). All of these factors influence both population regulation and the observed dispersion of parasites among hosts 
(Anderson \& Gordon 1982; Pacala \& Dobson 1988).

In the present paper, we examine two different types of data spanning a range of temporal and spatial scales for evidence of density-dependent effects that may contribute to population regulation of the ectoparasite Aponomma hydrosauri (Acari: Ixodidae), a tick species that infests reptiles. The first dataset is a series of rearing records for $A$. hydrosauri larvae, documenting that the rate of successful engorgement of larval ticks decreases with increasing tick burdens. This is direct evidence of density dependence at the scale of individual hosts. The second dataset represents 14 years of random captures of the main host of $A$. hydrosauri over $>40 \mathrm{~km}$ of road transects. The distribution of ticks among lizard hosts is generally negative binomial, and the aggregation coefficient $k$ increases with mean tick abundance. This relationship has been demonstrated to be a signal of density dependence in host-parasite populations (Pacala \& Dobson 1988), and it demonstrates that the density-dependent process observed in the rearing records is strong enough to have population level effects.

The indirect test for the presence of density-dependent mechanisms arose from interest in the often extreme overdispersion of parasites among hosts such that the variance in parasite load per host is much greater than the mean. This pattern has intrigued ecologists since Crofton (1971) first used the negative binomial distribution to quantify overdispersion. Anderson and Gordon (1982) elucidated the relationship between population dispersion and population dynamic processes of birth, death, immigration and emigration. They summarized their arguments with a "dispersion spectrum"
(Figure 1), using the variance-to-mean ratio as an indication of dispersion. In the absence of special processes, increasing the mean of a negative binomial distribution increases the dispersion, because the dispersion, as represented by the variance-to-mean ratio, is related to the mean $\mu$ and the aggregation coefficient $k$ by

$$
\frac{\sigma^{2}}{\mu}=1+\frac{\mu}{k}
$$

All else remaining equal, if $k$ increases, the dispersion will decrease, whereas if $\mu$ increases, the dispersion will increase.

The basis for interpreting changes in dispersion is a simple stochastic model where the number of parasites of a particular stage on a host is a balance between birth, death, immigration and emigration. Direct reproduction of the parasite within or on the host, heterogeneity in the rate of emigration off hosts, or the death rates of parasites within or on hosts will increase dispersion. These processes increase dispersion because they result in some hosts developing much greater parasite burdens than others. Parasite-induced mortality of hosts, or positive density dependence either in immigration onto hosts or on mortality rate of parasites on hosts, will reduce dispersion. These processes reduce dispersion by preferentially reducing parasite numbers on more heavily infected hosts. Therefore, density dependence in immigration or mortality rates is one possible explanation for dispersion remaining constant or reducing when density increases (Pacala \& Dobson 1988). Measuring changes in the aggregation coefficient $k$ was used to deduce the presence of density dependence in populations

\section{Dispersion Spectrum}

$\begin{array}{lll}s^{2} / x \rightarrow 0 & s^{2} / x=1 & s^{2} / x \rightarrow \infty \\ \begin{array}{l}\text { All hosts harbor } \\ \text { the same number } \\ \text { of parasites }\end{array} & \begin{array}{l}\text { Parasites } \\ \text { randomly } \\ \text { distributed }\end{array} & \begin{array}{l}\text { All hosts uninfected } \\ \text { except one which harbors } \\ \text { all the parasite population }\end{array}\end{array}$

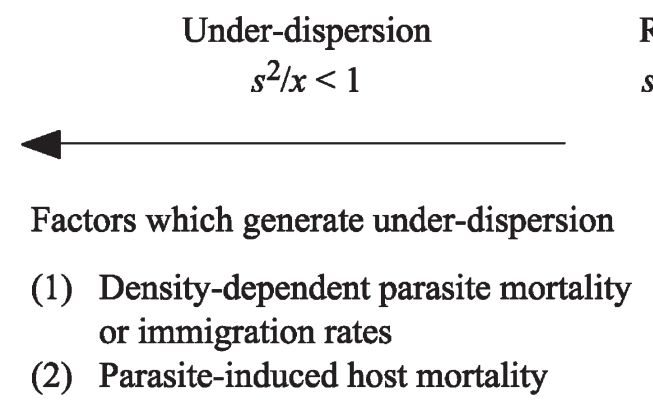

Random

$s^{2} / x=1$
Over-dispersion

$s^{2} / x>1$

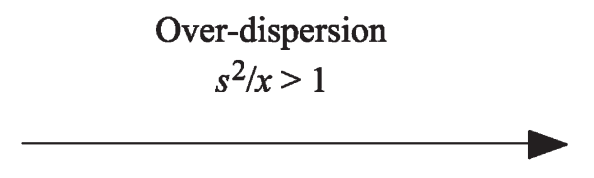

Factors which generate over-dispersion

(1) Direct reproduction of parasites within the host

(2) Heterogeneity between hosts in mortality or immigration rates of parasites

Figure 1. Dispersion spectrum for parasite distributions among hosts, indicating the processes that either increase or decrease dispersion. $s$ is the standard deviation of the parasite distribution, and $x$ is the mean. Adapted from Anderson and Gordon (1982). 
of two tick species Amblyomma dissimile and Amblyomma rotundatum on cane toads in South America (Lampo \& Bayliss 1996).

Anderson and Gordon's (1982) dispersion spectrum is a product of a static model that explicitly relates processes such as infection, survival on hosts, and reproduction to the parameters of a negative binomial distribution. Pacala and Dobson (1988) later extended this model. The most important conclusion from these models is that in the absence of density dependence in infection, survival or reproduction, the dispersion (i.e. the variance-to-mean ratio) of the parasite distribution increases with increasing mean density. If the negative binomial distribution is used to describe the distribution of parasites among hosts, then the absence of density dependence implies that $k$ will remain constant or decrease with increasing mean density, because this is the only way that the variance-to-mean ratio can increase. An increase in $k$ with average host density is therefore a signal that one or more processes in the parasite population are density dependent. We use $k$ as our indicator of density dependence rather than the variance-tomean ratio because we can estimate $k$ independently of the mean using maximum likelihood techniques. In addition, detecting a positive increase in $k$ is unambiguous, whereas a failure to detect an increase in the variance-to-mean ratio could arise through a lack of statistical power.

\section{Methods and Study Area}

\section{Study area and species}

The study area is mixed chenopod shrubland and mallee woodland near Mount Mary in the mid-north of South Australia. The region has an annual rainfall of approximately $250 \mathrm{~mm}$. Lizards were randomly encountered while walking or driving along road transects on 4-7 days each week from September until mid-December of each year. Aponomma hydrosauri is an ectoparasite of large reptiles in southern Australia. The predominant host is the sleepy lizard, Tiliqua rugosa. The study area straddles the biogeographical boundary of the distribution of A. hydrosauri; north of the boundary there are generally no $A$. hydrosauri ticks except for occasional outbreaks. The life cycle of $A$. hydrosauri has four stages: egg, larva, nymph, and adult (Sharrad 1979). It requires three hosts to complete its life cycle. Larvae, nymphs and adult females each attach to a host, engorge, and then detach (usually when the host is in an overnight refuge). Engorged larvae and nymphs molt to the next stage. Engorged females lay eggs that hatch into larvae. Unfed larvae, nymphs, and adults then wait in the refuge for a new host indi- vidual (or the same host) to attach to. Adult males take in small meals, and wait for long periods on the host where they mate with attaching females. Tick activity and development is confined to the spring and summer months when temperatures are warm and lizards are active (Bull \& Sharrad 1980).

\section{Methods}

\section{Detecting density dependence at the individual lizard scale}

From March 1983 to August 1985, records were kept of the number of larval ticks attached to captive lizards to obtain stocks of engorged larvae and unengorged nymphs for laboratory experiments. Fourteen T. rugosa were infested in this way with between 200 and 982 larvae of A. hydrosauri. Ticks were attached by placing a known number of larvae in a calico bag together with the host, and keeping them together in the dark for $24-48 \mathrm{~h}$ at $22{ }^{\circ} \mathrm{C}$. Lizards had their mouths taped so that ticks could not be consumed. The number of larvae successfully attaching was estimated by counting the ticks that remained in the bag after the host was removed.

Hosts were then maintained in a $25{ }^{\circ} \mathrm{C}$ room with a 12 : 12 photoperiod in individual cages with wire floors. A heat lamp above the cage allowed lizards to raise their body temperature to $30-35{ }^{\circ} \mathrm{C}$ during the light phase. Ticks that engorged and detached from the host fell through the floor onto a tray, where they were collected. For four of the 14 hosts, the proportion engorging was less than $1 \%$, more than 10 times less than that observed on other hosts, and statistical outliers by any measure used. These engorgement failures are either the result of unrecorded variations in experimental procedure, or caused by extreme between-host variations in suitability for ticks. Regardless of the reasons, these zero engorgement results clearly come from a different distribution from the remaining data, so these four hosts were removed from the data set before final analysis.

We used generalized linear modeling (GLM; McCullagh \& Nelder 1983) to analyze the probability of successful engorgement as a function of the number of attached larvae. Initially, we used a binomial error distribution with a logit link. This is analogous to a single generation key factor analysis (Varley et al. 1973), and is similar to the regression methods commonly employed by both advocates (e.g. Hassell et al. 1987) and critics (e.g. Strong 1989) of density dependence. However, preliminary analysis indicated that large amounts of extra-binomial variation were present. Such extra-binomial variation can arise from either within-host correlations in engorgement success or heterogeneity between hosts, and invalidates parameter standard errors 
and significance tests based on binomial error distributions (Collet 1991). Therefore we calculated bias-corrected and accelerated $\left(\mathrm{BC}_{a}\right)$ confidence intervals for the regression parameters from 5000 resamples with bootstrapping (Efron \& Tibshirani 1993) to estimate the magnitude of the density-dependent effect without making assumptions about the underlying distribution. Finally, we calculated the influence of each datum on the parameter estimates with a jackknife-after-bootstrap procedure, which provides a standardized measure of the influence of each point, and is analogous to Cook's distance (Anonymous 1999; Chernick 1999). Values of relative influence larger than two are of concern.

\section{Distribution of ticks among lizards}

The dataset used in the present paper consists of 14 years (1984-1997) of captures of sleepy lizards ( Tiliqua rugosa ) along six transects in the Mount Mary region,

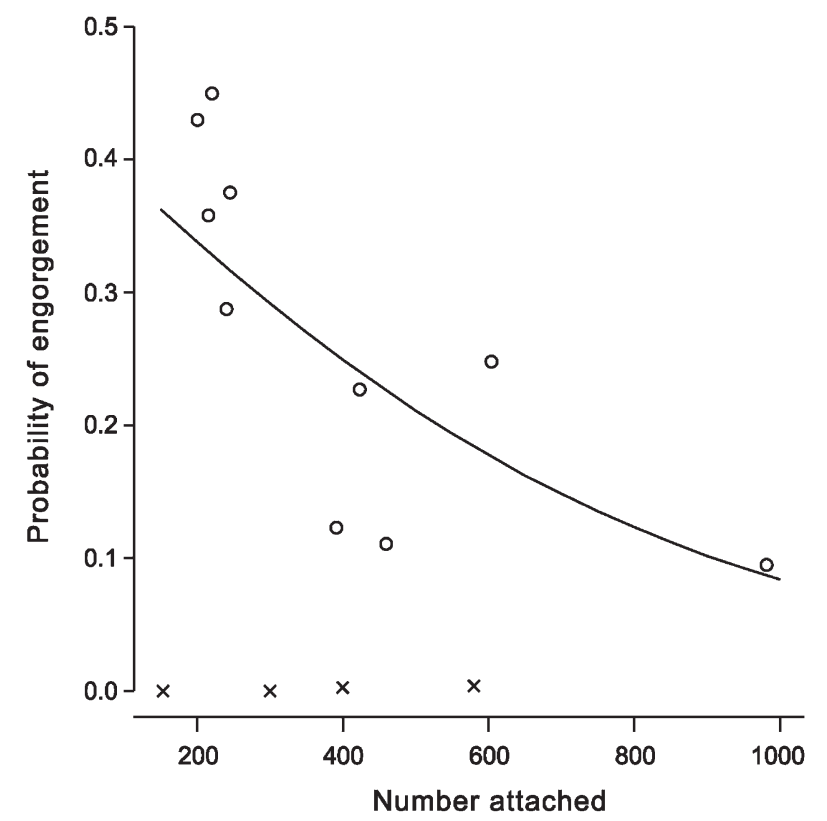

Figure 2. Proportion of successful engorging larvae as a function of the number of larvae attached to a single lizard. Each point is a different host. The hosts with less than $1 \%$ success are indicated with crosses; these hosts were not included in the final model fit. The solid line is the predicted probability from the quasi-likelihood estimate using only the points indicated with circles. at a spatial resolution of $50 \mathrm{~m}$ (see Bull 1995 for details). Each data point is a count of a number of ticks on a lizard at a point in time and space. Because larvae take 30 days on average to engorge, and because most tick records on lizards are of larvae, we considered that records more than 30 days apart were independent samples of tick burden. Where an individual lizard was captured more than once in a season, multiple captures were only included if they were 30 or more days apart. For shorter intervals between captures only the first record was included to reduce double-counting of ticks and distorting the frequency distributions of ticks among lizards.

There is considerable large-scale heterogeneity along transects in the abundance of $A$. hydrosauri as a result of the biogeographical boundary and variation in the amount of effort expended in searching for lizards. This results in transect sections where there are no or very few $A$. hydrosauri captured in some years for reasons completely unrelated to population regulation. We eliminated this large-scale heterogeneity to ensure that our results are based only on areas south of the biogeographical boundary that were well sampled. We divided each transect into 1-km blocks, and excluded blocks from the dataset with either less than 100 lizard captures over the duration of the study, or less than 0.25 A. hydrosauri per lizard over the duration of the study. There was a total of 7774 capture records in the final dataset.

We excluded male ticks from counts of adult ticks on lizards. Male ticks remain attached to hosts for extended periods of time, and therefore may bias the numeric results, when considering recaptures of the same individuals.

We estimated parameters for the negative binomial by minimizing the negative log likelihood $L$ (Hilborn \& Mangel 1997):

$$
\left.L(\mathbf{Y} m, k)=-\sum_{i=1}^{n} \ln \left(\frac{\Gamma(k+Y i}{\Gamma(k)+Y i !}\right)\left(\frac{k}{k+m}\right)^{k}\left(\frac{m}{m+k}\right)^{Y i}\right)
$$

where $\mathbf{Y}$ is the vector of $n$ observations of numbers of ticks on lizards, $m$ is the mean, and $k$ is the aggregation coefficient. The minimization was performed with the nlminb algorithm in S+ version 4.0. We checked that the negative binomial distribution fitted the data using Gtests (Sokal \& Rohlf 1981). We examined the relation-

Table 1. Bootstrap mean, standard error, and BCa confidence limits on the logistic regression parameters of the relationship between engorgement success and the number of ticks attached showing that increasing tick burdens always decrease the probability of successful engorgement (5000 bootstrap resamples were used).

\begin{tabular}{llllll}
\hline & & \multicolumn{4}{c}{ Confidence Limits } \\
\cline { 3 - 6 } Parameter & Mean (SE) & $2.5 \%$ & $5 \%$ & $95 \%$ & $97.5 \%$ \\
\hline Intercept & $-0.14(0.43)$ & -0.95 & -0.81 & 0.24 & 0.80 \\
No. attached & $-0.0025(0.001)$ & -0.0058 & -0.0042 & -0.0011 & -0.0008 \\
\hline
\end{tabular}


ship between the mean and the aggregation coefficient by calculating simple correlation coefficients for each life history stage.

\section{Results}

\section{Detecting density dependence at the individual lizard scale}

Observations of the proportion of tick larvae successfully engorging in the laboratory suggested both a density-dependent response at high numbers, and significant heterogeneity among hosts (Figure 2). After eliminating the four obvious outliers as discussed in the methods, we estimated the parameters of a binomial generalized linear model with logit link function as

$$
\begin{aligned}
& p\{\text { successful engorgement }\}=\frac{e^{\beta}}{1+e^{\beta}} \\
& \beta=-0.243-0.002 \text { (no. of ticks) }
\end{aligned}
$$

and the $\mathrm{BC}_{a}$ confidence intervals for the slope parameter do not include 0 at the $95 \%$ level (Table 1). In fact, only five of the 5000 bootstrap replicates were equal to or larger than 0 , the largest being 0.003 (Figure 3).

Particularly in light of the small sample size, it is useful to have an estimate of the influence of each datum on the parameter estimates. None of the 10 data points had a standardized influence larger than two in a jack-

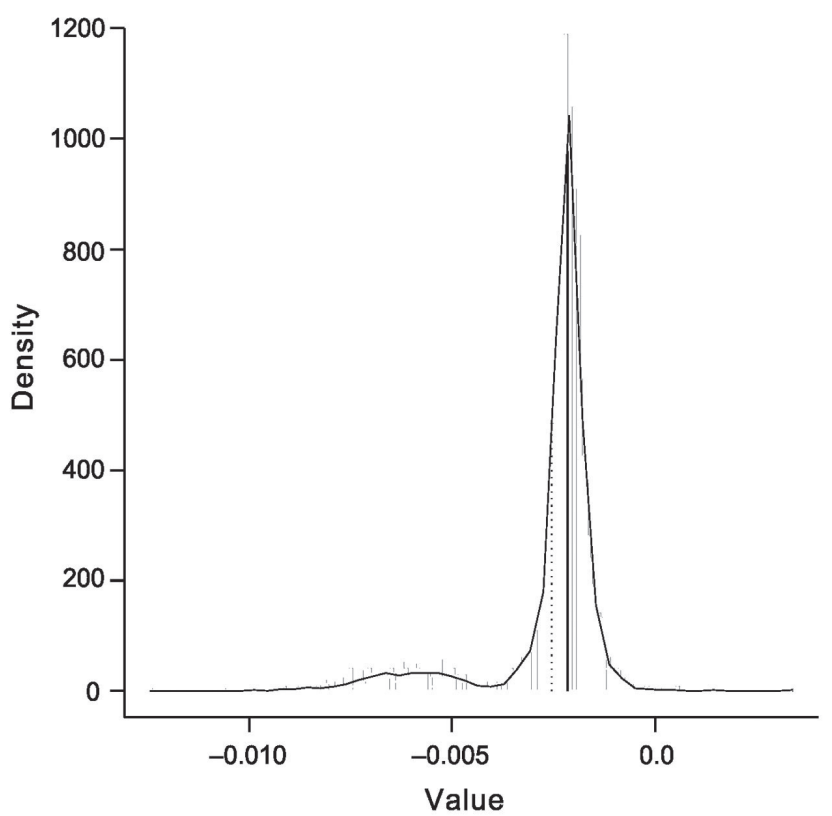

Figure 3. Kernel density plot of the 5000 bootstrap estimates of the slope parameter. The estimate from the observed dataset is shown using the solid vertical line, and the mean bootstrap estimate is the dashed vertical line. The bootstrap replicates range from -0.012 to 0.003 . knife-after-bootstrap procedure; however, data points with larger numbers of ticks had larger relative influences (Figure 4). When one of these points was present in the dataset, the bootstrap replicate slope tended towards zero, that is, these relatively influential points reduce the magnitude of the estimated density-dependent effect. Thus, although the dataset is small, if this causes the analysis to be biased, then it is biased against density dependence.

This analysis is direct evidence of density dependence in the rate of successful engorgement of tick larvae, a key process in the life history of this species. However, the mere existence of a density-dependent process does not imply that it contributes to population regulation. For that, we must look for effects of density dependence at the scale of the entire population by looking at changes in the tick distribution among hosts as tick abundance changes.

\section{Distribution of ticks among lizards}

In the reduced dataset, the maximum number of ticks observed on a single lizard was 1060, but $95 \%$ of lizards captured had 30 or fewer ticks. Less than $1 \%$ of captured lizards had more than 100 ticks.

We estimated the parameters (i.e. the mean $m$ and aggregation coefficient $k$ ) of the negative binomial distribution from all tick life history stages summed together within each year. These data fitted a negative binomial distribution in only one year. However, if the parame-

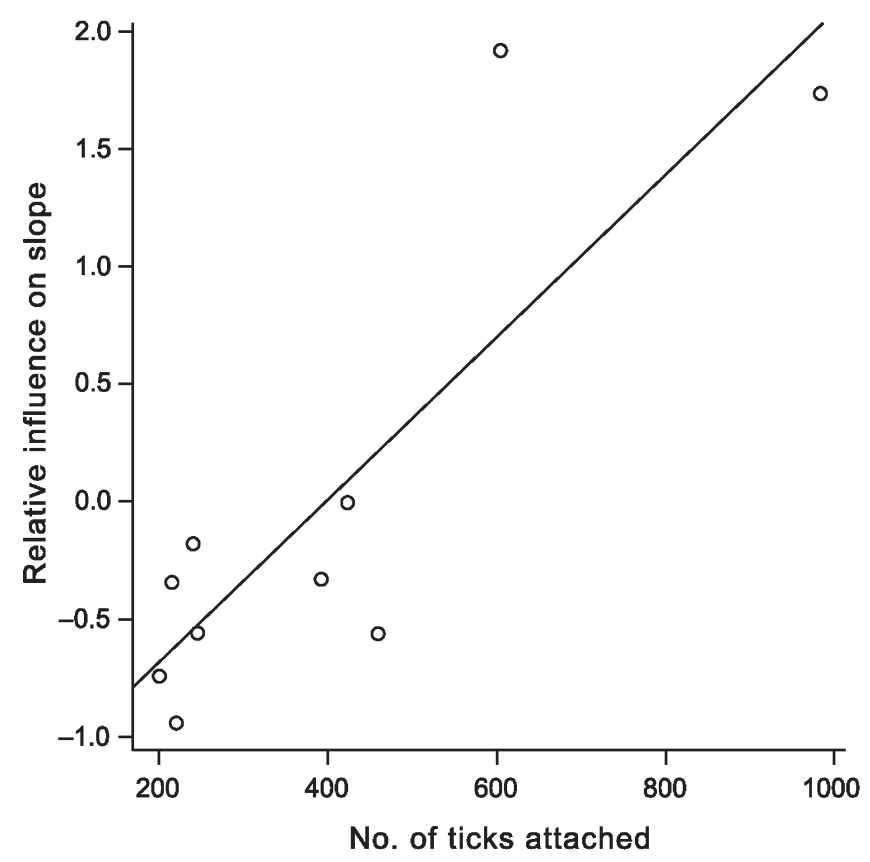

Figure 4. Relative influence of each point on the slope parameter as a function of the number of ticks attached. The solid line is a least squares fit to highlight the trend. 
ters were independently estimated for each of the three life history stages within each year, the data fitted a negative binomial distribution in most years. The distribution of adults fitted a negative binomial distribution in 13 out of 14 years, nymphs in 11 out of 14 years, and larvae in nine out of 14 years. One possible reason why larvae were less conforming may be that larvae do not board lizards independently of one another, because adult females deposit all of their eggs in a single lizard refuge. Therefore, a lizard is likely to encounter many larvae waiting for a host all at once. The number of processes (engorgement, detachment, molting, or waiting for a host) that separate individual nymphs and individual adults from each other means that nymphs and adults are more likely to be boarding hosts independently of one another.

For all three life history stages, the aggregation coefficient $k$ increases with increasing mean tick burden (Figure 5). Therefore the dispersion of the population is decreasing with increasing abundance, and this is evidence that one or more density-dependent processes are operating with sufficient strength to have an influence at the scale of the entire population.

\section{Discussion}

We have demonstrated that the probability of successful engorgement decreases with increasing tick burden, evidence that density dependence occurs in at least one key life history process of A. hydrosauri. Furthermore, we have shown that the dispersion of the entire tick population among the host population changes with increasing tick abundance in a manner consistent with densitydependent population regulation. Later in the present paper we will revisit each of these results and place it in a context of Anderson and Gordon's (1982) dispersion spectrum and Pacala and Dobson's (1988) model.

\section{Probability of engorgement decreases with increasing tick burden}

Ticks that fail to engorge die, so a decrease in engorgement success with density is a density-dependent increase in mortality of the parasite on the host. Increasing mortality with parasite abundance is one of the processes that can lead to a reduction in the dispersion of ticks among hosts (Figure 1). It is unclear whether this effect detected at the level of individual hosts can lead to population regulation, because the laboratory tick densities at which the experiment was carried out are rare in the field. If we use the model to extrapolate engorgement success down to smaller numbers, a larva on a lizard with 100 other ticks has a $39 \%$ chance of success compared with $44 \%$ for a larva alone. Tick burdens of 100 or greater occur less than $1 \%$ of the time in the field data set.
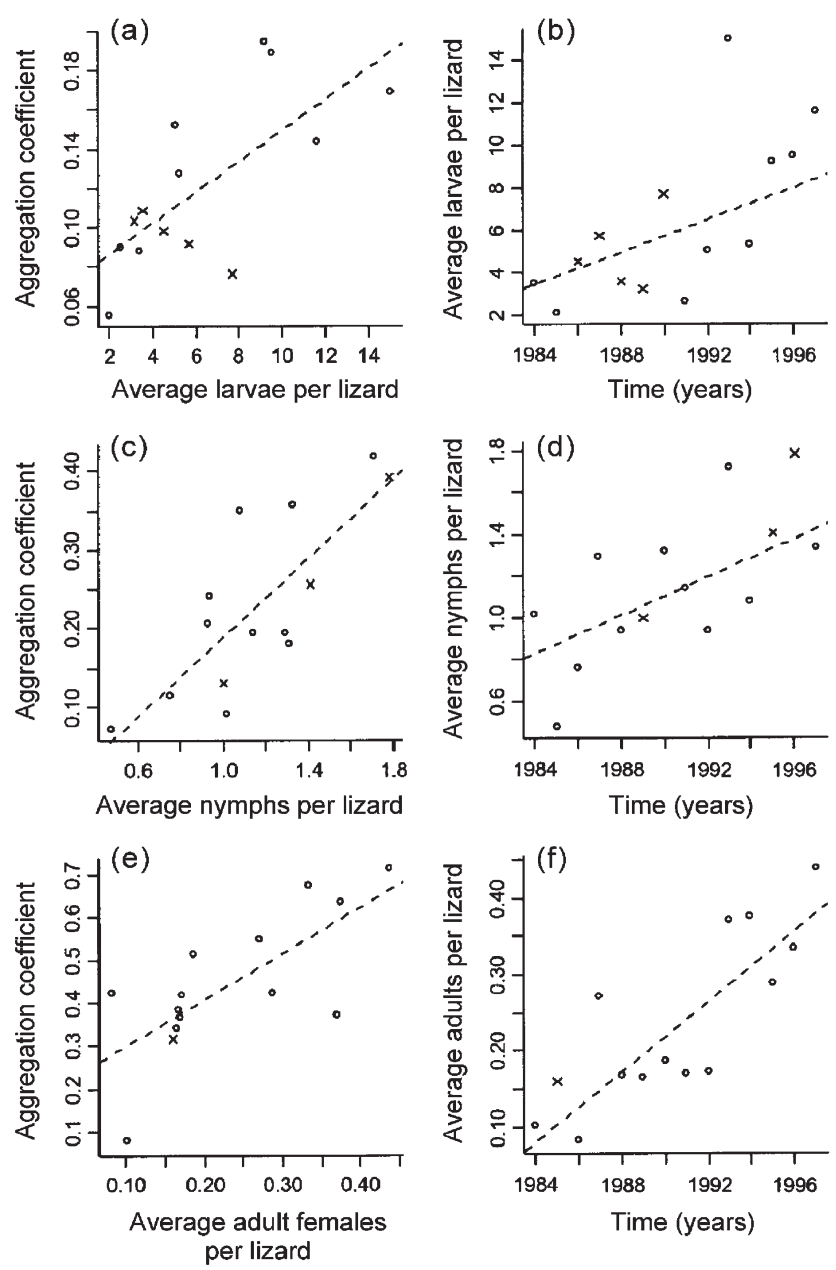

Figure 5. Plots of the average density versus (a, c, e) the aggregation coefficient $(k)$ or $(\mathbf{b}, \mathbf{d}, \mathbf{f})$ time for $(\mathbf{a}, \mathbf{b})$ larvae, $(\mathbf{c}$, d) nymphs, and $(\mathbf{e}, \mathbf{f})$ adults at the landscape scale. Points marked with $X$ do not fit a negative binomial distribution. Dashed lines are least squares fitted to highlight the trends. The correlations between the aggregation coefficient and average density are (a) $r=0.7$, (b) $r=0.78$, and (c) $r=0.72$. $P<0.01$ for all three correlations.

However, the present data may under-represent the potential for a density-dependent mechanism affecting larval engorgement success to regulate populations of A. hydrosauri. Bull et al. (1989) conducted a series of experiments to look for interspecific competition between A. hydrosauri and another reptile tick, Amblyomma limbatum. Two of the treatments had 100 and $300 \mathrm{~A}$. hydrosauri larvae on each host, and had average engorgement successes of $63 \%$ and $33 \%$, respectively. The GLM from our analysis predicts $39 \%$ and $30 \%$ for the same treatments. Within the range of the data used in the current study (200-960 ticks), the GLM appears to be consistent with the earlier experiment. However, the predicted engorgement success for a load of 100 ticks is considerably less than observed in Bull et al. (1989), suggesting that engorgement success rises considerably faster than predicted by the GLM at tick burdens less than 200 individ- 
uals. Therefore it is possible that the density-dependent effect is in fact much stronger at tick burdens commonly observed in the field.

It is unclear from these data what underlying mechanism is contributing to the observed reduction in success at high density. For example, if the reduction in success was caused by a limited number of attachment sites on a lizard, then the presence of ticks in any life stage at the time of attachment will influence the probability of success. Alternatively, the effect could take place after attachment, and could be related to host physiology or immunological responses. Higher tick density may induce a stronger defensive reaction from the host.

There are other possible density-dependent mechanisms that could regulate populations of A. hydrosauri. These include: density-dependent predation by ants in refuges, density-dependent engorgement success of nymphs or adults, and density-dependent effects of mate availability on fertilization of adult females. The frequency and magnitude of all these effects are unknown at present.

\section{Aggregation coefficient ( $k$ ) increases with increasing density}

Pacala and Dobson (1988) showed that a significant increase in $k$ with density among years was evidence for at least one of three processes operating: parasite-induced host mortality, density-dependent parasite mortality, and parasite-induced changes in a host's probability of infection (i.e. immigration rates onto hosts). There is no evidence that $A$. hydrosauri causes mortality in the host T. rugosa (Bull \& Burzacott 1993; Main \& Bull 2000), at least at tick burdens commonly observed in the field. Therefore parasite-induced host mortality is unlikely to contribute to an increase in $k$ with tick burdens. This suggests either that the mortality rate of parasites is density dependent, or that tick infections produce heterogeneity in the infection rate. Both of these possible mechanisms for the observed increase in $k$ in the field data were detected at the individual level in the laboratory attachment experiment. The first mechanism is the overall increase in mortality on hosts (decreased engorgement success) with increasing tick abundance. The second mechanism, heterogeneity among hosts in infection rates, could be responsible for the outlying responses (four hosts with zero engorgement success) and/or the high levels of extra-binomial variation in the number of ticks successfully engorging.

Density-dependent decreases in dispersion have been noted in other host-parasite systems (e.g. Scott 1987). Kretzschmar and Adler (1993) developed a general model of host-parasite dynamics that incorporated the effects of aggregation, and showed that a positive relationship between the mean density and an aggregation parameter stabilized the population dynamics. How- ever, their model made one critical assumption: namely that host mortality rates increase linearly with parasite burden. If host mortality rates do not increase with parasite burden, then the Kretzschmar and Adler model predicts that there will be no positive equilibrium, that is, the populations are completely unregulated. Our work here clearly shows that a density-dependent decrease in dispersion can arise without this assumption, and therefore their model may not apply to a broad range of systems.

In conclusion, we have detected density dependence in larval engorgement success through two independent sources of evidence at very different temporal scales. The debate on the extent to which natural populations are regulated through density-dependent mechanisms will continue. Our work is another piece of evidence showing that density-dependent mechanisms broadly contribute to population regulation in natural populations.

\section{Acknowledgments}

Australian Research Council Grants to C. Michael Bull supported this work over many years. Early versions of this manuscript benefited from comments by Mark Burgman, Leonore Fahrig, Hugh Possingham, and Hamish McCallum.

\section{References}

Anderson R. M. \& Gordon D. M. (1982) Processes influencing the distribution of parasite numbers within host populations with special emphasis on parasite-induced host mortalities. Parasitology 85, 373-98.

Anonymous (1999) S-Plus 2000 Guide to Statistics. MathSoft, Seattle.

Bonsall M. B., Jones T. H. \& Perry J. N. (1998) Determinants of dynamics: Population size, stability, and persistence. Trends Ecol. Evol. 13, 174-6.

Bull C. M. (1995) Population ecology of the sleepy lizard, Tiliqua rugosa, at Mt. Mary, South Australia. Aust. J. Ecol. 20, 393-402.

Bull C. M. \& Burzacott D. (1993) The impact of tick load on the fitness of their lizard hosts. Oecologia 96, 415-9.

Bull C. M., Burzacott D. \& Sharrad R. D. (1989) No competition for resources between two tick species at their parapatric boundary. Oecologia 79, 558-62.

Bull C. M. \& Sharrad R. D. (1980) Seasonal activity of the reptile tick, Aponomma hydrosauri (Acari: Ixodidae) in experimental enclosures. J. Aust. Entomol. Soc. 19, 47-52.

Chernick M. R. (1999) Bootstrap Methods: A Practicioner's Guide. John Wiley and Sons, New York.

Collet D. (1991) Modelling Binary Data. Chapman \& Hall, London.

Crofton H. D. (1971) A quantitative approach to parasitism. Parasitology 62, 179-94. 
den Boer P. J. (1991) Seeing the trees for the wood: Random walks or bounded fluctuations of population size. Oecologia 86, 484-91.

Efron B. \& Tibshirani R. J. (1993) An Introduction to the Bootstrap. Chapman \& Hall, New York.

Esch G. W. \& Fernández J. C. (1993) A Functional Biology of Parasitism: Ecological and Evolutionary Implications. Chapman \& Hall, London.

Hassell M. P., Southwood T. R. E. \& Reader P. M. (1987) The dynamics of the viburnum whitefly (Aleurotrachelus jelinekii): A case study of population regulation. J. Anim. Ecol. 56, 283-300.

Hilborn R. \& Mangel M. (1997) The Ecological Detective: Confronting Models with Data. Princeton University Press, Princeton.

Kretzschmar M. \& Adler F. R. (1993) Aggregated distributions in models for patchy populations. Theor. Popul. Biol. 43, 1-30.

Lampo M. \& Bayliss P. (1996) The impact of ticks on Bufo marinus from native habitats. Parasitology 113, 199-206.

McCullagh P. \& Nelder J. A. (1983) Generalized Linear Models. Chapman \& Hall, London.

Main A. R. \& Bull C. M. (2000) The impact of tick parasites on the behaviour of the lizard Tiliqua rugosa. Oecologia 122, 574-81.

Murray B. J. (2000) Dynamics of an age-structured population drawn from a random numbers table. Austral Ecol. 25, 297-304.

Pacala S. W. \& Dobson A. P. (1988) The relation between the number of parasites host and host age: Population-dynamic causes and maximum-likelihood estimation. Parasitology 96, 197-210.
Peet R. K. (1991) Lessons from nature: Case studies in natural systems. In: Foundations of Ecology: Classic Papers with Commentaries (eds. L. A. Real \& J. A. Brown) pp. 605-15. University of Chicago Press, Chicago.

Ray C. \& Hastings A. (1996) Density dependence: Are we searching at the wrong spatial scale? J. Anim. Ecol. 65, 556-66.

Roitberg B. D., Mangel M. \& Tourigny G. (1990) The density dependence of parasitism by tephritid fruit flies. Ecology 71, 1871-85.

Scott M. E. (1987) Temporal changes in aggregation: A laboratory study. Parasitology 94, 583-95.

Sharrad R. D. (1979) Studies of the Factors Which Determine the Distribution of Three Species of South Australian Ticks. University of Adelaide, Adelaide.

Smith G. (1994) Parasite population density is regulated. In: Parasitic and Infectious Diseases: Epidemiology and Ecology (eds. M. E. Scott \& G. Smith), pp. 47-64. Academic Press, San Diego.

Sokal R. R. \& Rohlf F. J. (1981) Biometry. W. H. Freeman, New York.

Stiling P., Throckmorton A., Silvanima J. \& Strong D. R. (1991) Does spatial scale affect the incidence of density dependence? A field test with insect parasitoids. Ecology 72, 2143-54.

Strong D. R. (1989) Density independence in space and inconsistent temporal relationships for host mortality caused by a fairyfly parasitoid. J. Anim. Ecol. 58, 1065-76.

Tyre A. J. \& Tenhumberg B. (2000) Hidden mechanisms generate negative feedbacks in a stochastic model: A comment on Murray. Austral Ecol. 25, 305-7.

Varley G. C., Gradwell G. R. \& Hassell M. P. (1973) Insect Population Ecology. Blackwell, Oxford. 